\title{
Characterization of Lateral Root Development at the Onset of Storage Root Initiation in 'Beauregard' Sweetpotato Adventitious Roots
}

\author{
Arthur Villordon ${ }^{1}$ \\ LSU AgCenter Sweet Potato Research Station, 130 Sweet Potato Road, Chase, \\ LA 71324
}

Don LaBonte and Julio Solis

LSU AgCenter School of Plant, Environmental, and Soil Sciences, 137 J.C. Miller Hall, Baton Rouge, LA 70803-2120

\section{Nurit Firon \\ Institute of Plant Sciences, The Volcani Center, Agricultural Research Organization, P.O. Box 6, Bet Dagan, 50250, Israel}

Additional index words. Ipomoea batatas, lateral root density, anomalous cambium, pencil roots, lignified roots

\begin{abstract}
This study characterized lateral root (LR) development attributes during the onset of storage root (SR) initiation stage in 'Beauregard' sweetpotato. SR initiation has been defined as the appearance of cambia around the protoxylem and secondary xylem elements. Our results showed that 20-day-old adventitious roots (ARs) classified as SRs had $53 \%$ and $85 \%$ greater mean LR count than pencil roots (PRs) and lignified roots (LGs), respectively. SRs had $53 \%$ and $78 \%$ greater mean LR density relative to PRs and LGs, respectively. SRs had $66 \%$ and $130 \%$ greater mean total LR length than PRs and LGs, respectively. SRs had lower mean main root (MR)/LR length ratio compared with PRs $(-38 \%)$ and LGs $(-60 \%)$. SRs had $70 \%$ and $134 \%$ greater mean surface area than PRs and LGs, respectively. SRs had lower mean MR/LR surface area ratio compared with PRs $(-42 \%)$ and LGs $(-62 \%)$. The plot of the first and second principal components revealed the presence of a gradient between extreme LG and SR clusters, suggesting a developmental transition between LGs and SRs with PRs representing an intermediate developmental stage. Although AR architecture is not the sole determinant of SR formation, our data help provide a basis for integrating $A R$ architecture attributes with other factors that are known to influence SR initiation. Growth substrate moisture variability influenced LR development during the critical SR initiation period. Relative to the control treatments, water deprivation 10 to 20 days after transplanting (DAT) reduced mean LR count, length, and surface area by $49 \%, 103 \%$, and $94 \%$, respectively. Saturated conditions 10 to 20 DAT reduced mean LR count, length, and surface area by $75 \%, 81 \%$, and $77 \%$, respectively. These results represent the first evidence for the association between anatomical cues of SR initiation and root architecture and provide corroborating data that soil moisture variability 10 to 20 DAT directly influences SR yield potential through AR architecture modifications that are associated with diminished SR formation. This information can be used to further optimize SR yield by identifying agroclimatic and management variables that are associated with desirable LR development during the critical SR initiation stage.
\end{abstract}

Koshimizu and Nishida (1949) measured LR branching in sweetpotatoes and postulated that LR branching may be very important "to supply the internal growth elements" for storage root formation. Pardales and Yamauchi (2003) studied sweetpotato LR development during the establishment period, which they defined as $\approx 4$ weeks after transplanting and suggested a link between LR variability and the onset of SR initiation in ARs. Recent molecular data have provided further evidence for the presumptive link between LR development and SR initiation. Specifically, IbMADS1 has been documented as related to SR initiation in sweetpotato $(\mathrm{Ku}$ et al., 2008). They have shown that IbMADS1 transcripts were localized to emerging LR primordia and immature meristematic cells such as the protoxylem and protophloem within the AR stele ( $\mathrm{Ku}$ et al., 2008). SR initiation has previously been defined as the appearance of cambia around the protoxylem and secondary xylem elements (Togari, 1950; Wilson and Lowe, 1973). Togari (1950) also suggested that once stele lignification occurred, SR initiation did not proceed. LRs originate from the pericycle and the radial location of LRs is correlated with the internal architecture of the stele (Laskowski et al., 1995). In radish (Raphanus sativus) and Arabidopsis, which have diarch steles, LRs arise from pericycle cells located in files adjacent to a xylem pole (Laskowski et al., 1995). Thus, in sweetpotato ARs, the number of LR rows corresponds to the number of protoxylem poles (Yasui, 1944). In 'Beauregard' sweetpotato, the number of protoxylem poles varies from five to 10 , but the majority of ARs show almost equal occurrence of five (pentarch) or six (hexarch) poles (Villordon et al., 2009a). Previous reports of the morphological and anatomical characterization of SR initiation did not provide LR developmental data (Belehu et al., 2004; Togari, 1950; Villordon et al., 2009a; Wilson and Lowe, 1973). Pardales and Yamauchi (2003) quantified LR count and total length response to soil moisture variability among greenhousegrown sweetpotato genotypes but did not present data for SR initiation. Recent data have showed that LR initiation and development were detected as early as 9 DAT among in situ field-grown 'Beauregard' plants (Villordon et al., 2011). We have previously reported that SR initiation was detected as early as $13 \mathrm{~d}$ in field-grown 'Beauregard' plants (Villordon et al., 2009b). In model species, LRs have been referred to as a major determinant of root architecture and contribute to water use efficiency and facilitate the extraction of micro- and macronutrients from the soil (Casimiro et al., 2003). Thus, the presumptive role of LR development in SR initiation represents a knowledge gap in the current research on internal and external factors that influence SR initiation.

The knowledge of the association between root architecture and SR initiation will benefit current and future studies in further understanding the mechanism of SR initiation in sweetpotatoes. Such information will also lead to further clarity in understanding how external stimuli such as soil moisture variability and nutrient availability promote or hinder SR initiation and can lead to modifications or enhancements of current management practices. Togari (1950) has previously reported that the growing environment during the first $20 \mathrm{~d}$ of AR development directly influenced SR initiation. Togari (1950) also reported that "dryness" and "shortage of $\mathrm{O}_{2}$ " during the first $20 \mathrm{~d}$ favored lignification and the development of PRs. Pardales and Yamauchi (2003) have previously documented that five sweetpotato genotypes (PRS118, PRS-74, PRS-46, VSP-4, and VSP-2) subjected to continuous deficient soil moisture showed an overall reduction in LR number and total length. Pardales and Yamauchi (2003) also reported that root development was suppressed among plants subjected to continuous "waterlogged" conditions. In a previous 32-d destructive sampling study, we have documented that the majority of greenhousegrown 'Beauregard' AR samples showed evidence for SR initiation at $19 \mathrm{~d}$ (Villordon et al., 2009b). The primary objective of this work was to provide quantitative morphological evidence of the link between LR development, a major determinant of root architecture, and anatomical cues associated with the onset of SR initiation stage in 'Beauregard' sweetpotato. A secondary objective was to document 
the relationship between soil moisture variability and LR development during the first $20 \mathrm{~d}$ of growth.

\section{Materials and Methods}

Plant materials. The greenhouse experiments were conducted in Chase, LA (lat. $32^{\circ} 6^{\prime} \mathrm{N}$, long. $91^{\circ} 42^{\prime} \mathrm{W}$ ). In each of 15 Oct. 2010 and 1 Oct. 2011, virus-tested 'Beauregard' Generation 1 SRs were bedded in washed river sand and served as a source of transplants. One set of experiments generated samples for anatomical observations and associating LR attribute with anatomical cues of SR initiation. The second set of experiments investigated the influence of growth substrate moisture content on LR development. Washed river sand was used as a growth substrate for all experiments. In each experiment, cuttings were set in 10-cm-diameter polyvinyl chloride $(\mathrm{PVC})$ pots $($ height $=30 \mathrm{~cm})$ with detachable plastic bottoms. Each plastic bottom had five drain holes ( $2 \mathrm{~mm}$ in diameter). In addition, each PVC pot had four rows of side drain holes $(2 \mathrm{~mm}$ in diameter; $3 \mathrm{~cm}$ apart within row) that were located diametrically opposite each other. Fertilizer application consisted of $0.76 \mathrm{~g}$ of $5 \mathrm{~N}-20 \mathrm{P}-$ $20 \mathrm{~K}$ per pot. The greenhouse temperature regime was $29^{\circ} \mathrm{C}$ for $14 \mathrm{~h}$ (day) and $18{ }^{\circ} \mathrm{C}$ for $10 \mathrm{~h}$ (night). Photosynthetic photon flux $(P P F)$ for all experiments ranged from 150 to $1300 \mu \mathrm{mol} \cdot \mathrm{m}^{-2} \cdot \mathrm{s}^{-1}$. Supplementary lighting was provided using white fluorescent lights $\left(\approx 42 \mu \mathrm{mol} \cdot \mathrm{m}^{-2} \cdot \mathrm{s}^{-1} P P F\right)$ for $14 \mathrm{~h} \cdot \mathrm{d}^{-1}$. $P P F$ was measured at the canopy level with a quantum sensor (Model QSO-S; Decagon Devices Inc., Pullman, WA). The relative humidity (RH) averaged $60 \%$. Temperature and $\mathrm{RH}$ were monitored at the canopy level using an integrated temperature and $\mathrm{RH}$ sensor (Model RHT; Decagon Devices Inc.). Unless otherwise indicated, the moisture of the growing substrate was maintained $\approx 50 \%$ of field capacity $[\approx 7 \%$ volumetric water content (VWC)]. Growth substrate moisture was measured with $\mathrm{ECH}_{2} \mathrm{O}$ soil moisture sensors (Model EC-5; Decagon Devices

Received for publication 25 Jan. 2012. Accepted for publication 14 May 2012.

Portions of this research were supported by Research Grant No. US-4015-07 from BARD, the U.S.-Israel Binational Agricultural Research and Development, USDA NIFA SCRI 2009-5118106071, and the Louisiana Sweetpotato Advertising and Development Fund.

We thank anonymous reviewers for valuable feedback and constructive comments.

Approved for publication by the Director of the Louisiana Agricultural Experiment Station as manuscript number 2012-260-6734.

Mention of trademark, proprietary product or method, and vendor does not imply endorsement by the Louisiana State University Agricultural Center nor its approval to the exclusion of other suitable products or vendors.

${ }^{1}$ To whom reprint requests should be addressed; e-mail avillordon@agcenter.lsu.edu.
Inc.) inserted vertically at the 2- to 7-cm depth. These growth conditions were very similar to those used in previous experiments that characterized AR development and the timing of SR initiation in 'Beauregard' sweetpotato (Villordon et al., 2009a, 2009b). In experiments that studied the effect of moisture levels on LR development, a uniform watering regime was imposed during the first $10 \mathrm{~d}$. This was to ensure that all cuttings were established (initiated ARs) and that ARs underwent initial LR development. After $10 \mathrm{~d}$, the following moisture treatments were imposed for an additional $10 \mathrm{~d}$ : control (moisture was maintained near $50 \%$ of field capacity; $\approx 200 \mathrm{~mL}$ distilled water per pot per irrigation event, up to three irrigation events until harvest), dry (water deprivation 10 DAT), and saturated (400 $\mathrm{mL}$ water per irrigation event, up to five irrigation events until harvest). Growth substrate moisture readings for the control treatment ranged from $5 \%$ to $8 \%$ VWC. The ranges for dry and saturated treatments were $1 \%$ to $5 \%$ VWC and $8 \%$ to $12 \%$ VWC, respectively. All sensors were connected to a data logger that was configured to collect measurements every $60 \mathrm{~min}$ (Model EM50R; Decagon Devices Inc.).

In all experiments, plants were grown for $20 \mathrm{~d}$ after which near-intact root systems were collected. At harvest, the detachable plastic bottoms were removed and the pot was tilted and the growth substrate was gradually removed using a stream of water. The planting dates for AR anatomical and LR attribute characterization experiments were 2 Mar. 2011, 12 Nov., 2011, and 28 Nov. 2011. The harvest dates were 22 Mar. 2011, 2 Dec. 2011, and 18 Dec. 2011. The planting dates for the growth substrate moisture experiments were 13 Apr. 2011 and 19 Dec. 2011. The harvest dates were 3 May 2011 and 8 Jan. 2012. All experiments were arranged as a randomized complete block repeated across planting dates. There were five replicates (one plant per pot $=$ one replicate) in each of the LR characterization experiments and in each of the substrate moisture experimental treatments. As a result of the variability of the number of adventitious roots per plant, there were unequal subsample sizes of adventitious roots that met the criteria for scanning and analysis in all experiments. In

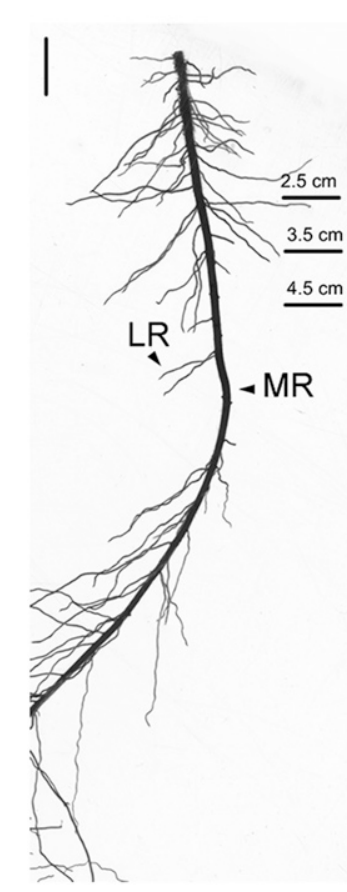

A

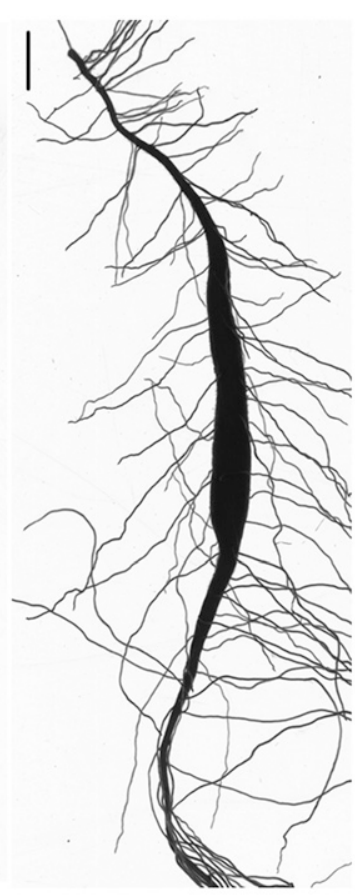

B

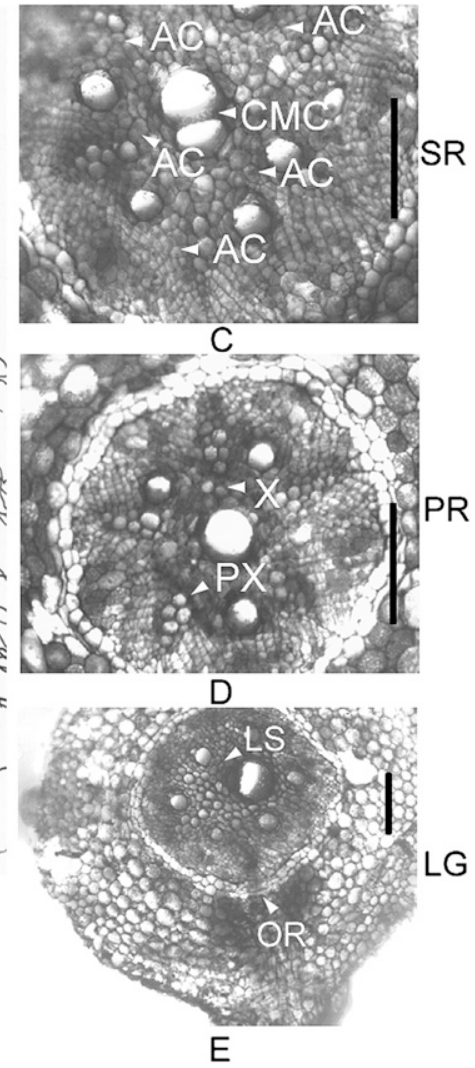

Fig. 1. Adventitious root samples harvested at 20 (A) and 40 (B) d after transplanting and representative micrographs used to classify 20 -d-old adventitious roots into various stages in 'Beauregard' sweetpotato: initiated storage root $(\mathbf{C})$, pencil $\operatorname{root}(\mathbf{D})$, and lignified $\operatorname{root}(\mathbf{E})$. Adventitious roots $(\mathbf{A}-\mathbf{B})$ were floated on waterproof trays and images were acquired using a scanner equipped with the WinRhizo positioning system. (A) Location of tissue sections used for anatomical examination for each 20-d-old adventitious root sample. Samples $\mathbf{C}-\mathbf{E}$ were derived from the $4.5-\mathrm{cm}$ section of the respective 20-dold adventitious root specimens. $\mathrm{LR}=$ lateral root; $\mathrm{MR}=$ main root; $\mathrm{SR}=$ initiated storage root; $\mathrm{PR}=$ pencil root; $\mathrm{LG}=$ lignified root; $\mathrm{AC}=$ anomalous cambium; $\mathrm{PX}=$ protoxylem; $\mathrm{CMC}=$ central metaxylem cell; $\mathrm{X}=$ protoxylem element remains connected to $\mathrm{CMC} ; \mathrm{LS}=$ lignified stele; $\mathrm{OR}=$ origin of lateral root. Scale bars for $\mathbf{A}-\mathbf{B}=1 \mathrm{~cm}$. Scale bars for $\mathbf{C}-\mathbf{E}=0.15 \mathrm{~mm}$. 
each experiment, extra replicates were grown for validation of visible storage root formation at $40 \mathrm{~d}$.

Root image acquisition and analysis. Preliminary calibration experiments were performed to determine optimal settings for image acquisition and analysis parameters. These experiments included comparison of scanner-based and manual measurements using representative AR samples. Based on these preliminary experiments, the image acquisition parameter was set to "medium" accuracy (400 dpi; image size $\approx 8 \mathrm{MB}$ ), whereas analysis precision was set to "high." In all experiments, intact ARs that were $20 \mathrm{~cm}$ or greater in length were floated on waterproof trays and scanned using a specialized Dual Scan optical scanner (Regent Instruments Inc., Quebec, Canada). The acquisition and image analysis software was WinRHIZO Pro (Version 2009c; Regent Instruments Inc.). Debris removal among scanned images was performed manually using the WinRhizo Pro Edition working mode. Debris consisted mainly of images of sand particles and occasional broken root segments $(1 \mathrm{~cm}$ or less in length). Root types were automatically classified based on root diameter, which was in turn based on predetermined size intervals. In our work, the intervals were set to 0 to 0.9 and 0.9 to $20 \mathrm{~mm}$. The first interval classified LRs, whereas the second interval classified the main AR (main root). These intervals were based on prior calibration experiments on 'Beauregard' ARs. Wilson and Lowe (1973) have previously reported that around the onset of SR initiation, the MRs were $\approx 1 \mathrm{~mm}$. LR attributes that were measured from scanned images included LR count, LR length (total length of all LRs), and LR surface area (total surface area of all LRs). MR length was verified using ImageTool (Univ. of Texas Health Science Center at San Antonio, available at ftp://maxrad6. uthscsa.edu). LR density was calculated by dividing LR count by MR length. The following ratios were calculated: MR length/LR length (MR/LR length ratio) and MR surface area/LR surface area (MR/LR surface area ratio).

Anatomical observations. AR samples for anatomical observations were prepared using free-hand sectioning and staining with toluidine blue (Eguchi and Yoshida, 2008). Samples were prepared by placing a proximal segment of an $\mathrm{AR}(\approx 7$ to $10 \mathrm{~cm})$ in a petri dish containing distilled water followed by the excision and staining of three separate tissue sections $(2.5,3.5$, and $4.5 \mathrm{~cm}$ from the proximal end) (Fig. 1A). The thickness of transverse sections ranged from 8 to $15 \mu \mathrm{m}$. Digital micrographs were taken with a Motic Cam 1000 (Motic Instruments Inc., British Columbia, Canada). In previous SR initiation studies, we routinely observed that anatomical cues of SR initiation (anomalous cambia) occurred within the 2.5 to $5 \mathrm{~cm}$ proximal end of an AR and this section typically became swollen after $30 \mathrm{~d}$ when grown in conditions described previously (Fig. 1B) (Villordon et al., 2009a, 2009b). Wilson and Lowe (1973) documented that this zone of thickening initially did not extend over the entire region of initiation.

Classification of adventitious root development stage. We used the criteria described by Wilson and Lowe (1973) in classifying the AR developmental stage: initiated SRs, PRs, and LGs. According to this scheme, ARs were classified as initiated when circular anomalous cambia were detected around the central metaxylem cell as well as around each of the discrete protoxylem elements in one or all of the proximal sections (Fig. 1C). For initiated ARs without a central metaxylem cell, primary cambia were associated with meristematic activity in the pith cells and the formation of anomalous cambia around the protoxylem elements. PRs were ARs that showed some meristematic activity around the central metaxylem cell, but one or more of the protoxylem elements remained connected to the central cell (Fig. 1D). LGs were ARs that eventually developed into mature thickened roots (up to $5 \mathrm{~mm}$ in diameter) with a heavily lignified stele, xylem rays, a broad secondary cortex, and limited secondary phloem (Fig. 1E).
Data collection and statistical analysis. A total of 106 ARs from three anatomical characterization experiments were unambiguously classified as SR $(\mathrm{n}=25), \mathrm{PR}(\mathrm{n}=32)$, and LG $(n=49)$. LR attribute data from these experiments were pooled and the combined data set was used for LR development attribute analysis $(\mathrm{n}=106$; AR anatomical characterization data set). Similarly, LR attribute data from the two growth substrate moisture experiments were pooled $(\mathrm{n}=136$; substrate moisture data set). The number of ARs that met the criteria for data acquisition varied among the moisture treatments: control ( $\mathrm{n}=$ $42)$, dry $(n=39)$, and saturated $(n=55)$. The number of ARs per plant ranged from two to eight. All numeric data were transformed using $\log 10$ and square root transformation, respectively. Ratios were arcsine-transformed. The unbalanced data sets were analyzed using SAS Proc Mixed (SAS Version 9.1; SAS Inc., Cary, NC). Fisher's least significant difference test at the $0.05 P$ level indicated that main effects were statistically significant for each of the AR anatomical characterization and substrate moisture data sets. The data presented were means and confidence intervals

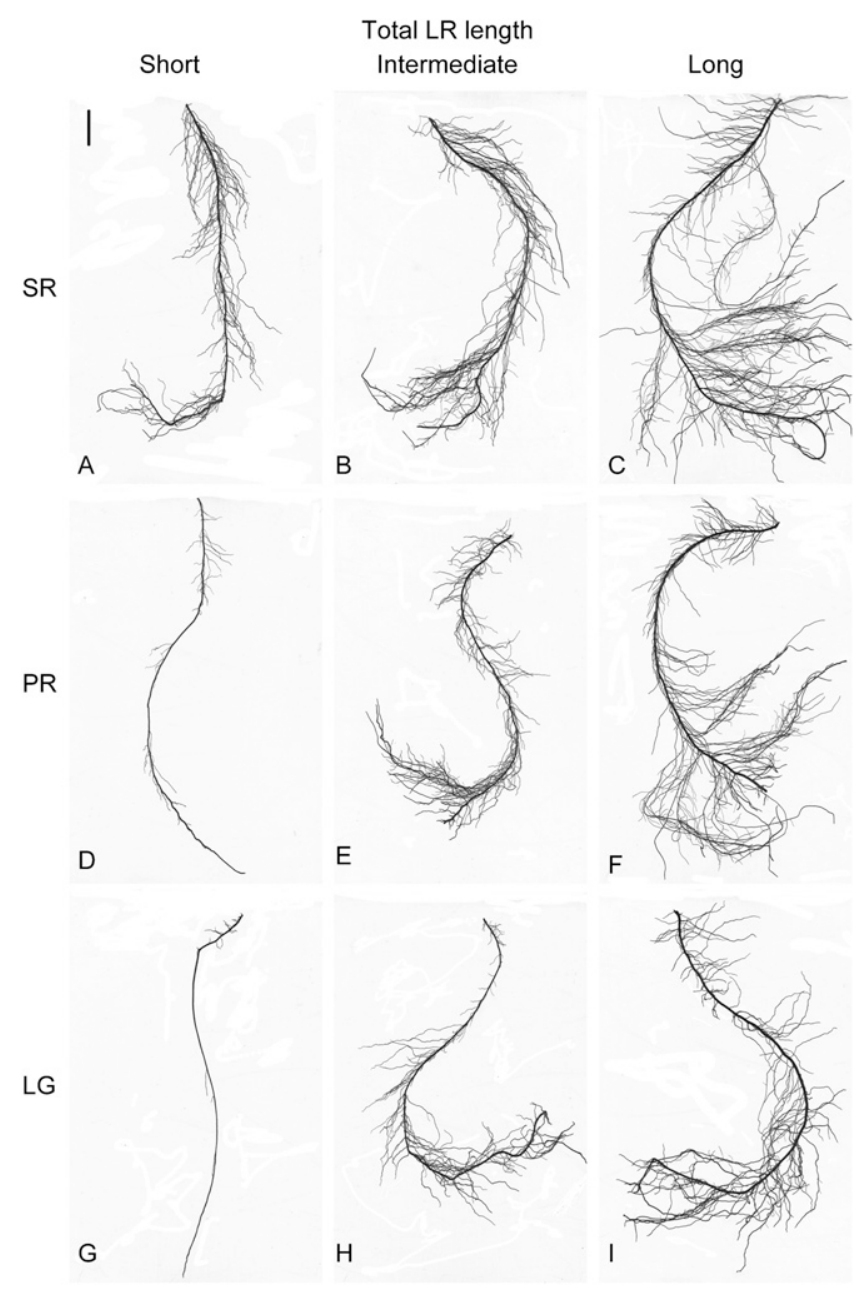

Fig. 2. Adventitious roots that represent the shortest, intermediate, and longest cumulative lateral root length for each of the following stages in 'Beauregard' sweetpotato: initiated storage root (SR), pencil $\operatorname{root}(\mathrm{PR})$, and lignified root $(\mathrm{LG}) . \mathrm{LR}=$ lateral root. Scale $\mathrm{bar}=2 \mathrm{~cm}$. 

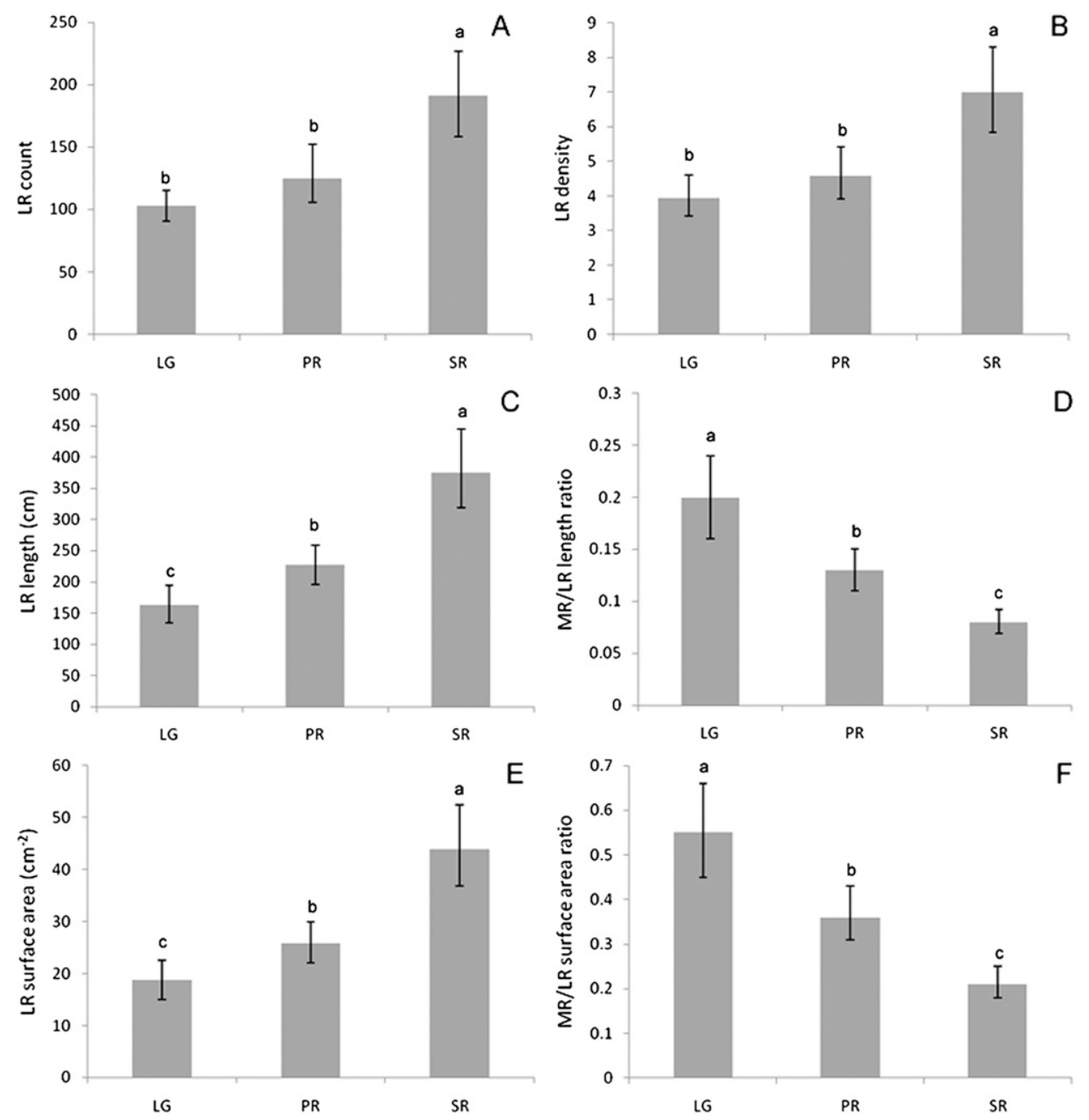

Fig. 3. Some statistical descriptive data of lateral root attributes of 'Beauregard' sweetpotato adventitious roots sampled at $20 \mathrm{~d}$ and classified into various stages: storage root (SR), pencil root (PR), and lignified root (LG). $\mathrm{LR}=$ lateral root; $\mathrm{MR}=$ main root. Bars represent means \pm bootstrapped confidence intervals $(95 \%$ confidence level). Mean comparisons were performed on transformed data: square-transformed (measurements), log 10 transformed (counts), and arcsinetransformed (ratios). Corresponding non-transformed values are shown. Columns with different letters differ significantly at the 5\% level by Fisher's least significant difference.

Table 1. Eigenvectors and eigenvalues for the first six principal components estimated from six lateral root attributes of 'Beauregard' sweetpotato adventitious roots grown in Louisiana. ${ }^{\mathrm{z}}$

\begin{tabular}{lrrrrrr}
\hline LR attributes & \multicolumn{1}{c}{ PC 1 } & \multicolumn{1}{c}{ PC 2 } & \multicolumn{1}{c}{ PC 3 } & \multicolumn{1}{c}{ PC 4 } & \multicolumn{1}{c}{ PC 5 } & \multicolumn{1}{c}{ PC 6 } \\
\hline Length & 0.432369 & -0.041885 & 0.447946 & 0.280676 & -0.261731 & 0.680709 \\
MR/LR length ratio & -0.410678 & 0.421374 & 0.260281 & -0.266416 & 0.564967 & 0.442579 \\
Surface area & 0.430035 & -0.027707 & 0.500011 & 0.188038 & 0.557471 & -0.467076 \\
MR/LR area ratio & -0.396377 & 0.512477 & 0.259509 & 0.592851 & -0.312789 & -0.252186 \\
Count & 0.384339 & 0.593236 & 0.127786 & -0.573943 & -0.345425 & -0.187873 \\
Density & 0.393242 & 0.453167 & -0.630804 & 0.366063 & 0.290483 & 0.153964 \\
Eigenvalue & 5.136068 & 0.642359 & 0.171238 & 0.031302 & 0.017535 & 0.001494 \\
Cumulative & 0.856 & 0.9631 & 0.9916 & 0.9968 & 0.9998 & 1.0000 \\
\hline
\end{tabular}

${ }^{\mathrm{z}} \mathrm{LR}=$ lateral root; $\mathrm{MR}=$ main root; $\mathrm{PC}=$ principal component. Principal component analysis was performed on standardized LR attribute data (subtraction of mean followed by division by SD). Description of experimental procedures is found in "Materials and Methods."

calculated from balanced data sets $(n=60)$ that were generated by stratified resampling in IBM SPSS Statistics (Version 19; SPSS Inc., Chicago, IL). Bootstrapped confidence intervals were presented for all LR attribute data. Principal components analysis (PCA) (type $=$ correlation) was performed on the balanced AR anatomical characterization data set using SAS PRINCOMP (SAS Version 9.1; SAS Inc.)

\section{Results}

Relationship between lateral root development and adventitious root stage. Representative images of LR development representing shortest, intermediate, and longest total LR lengths for each of SR, PR, and LG stages are shown in Figure 2. ARs classified as SRs had $53 \%$ and $85 \%$ greater mean LR count than PRs and LGs, respectively
(Fig. 3A). SRs had 53\% and 78\% greater mean LR density than PRs and LGs, respectively (Fig. 3B). There were no significant differences in mean LR count and density between PRs and LGs. SRs had 66\% and $130 \%$ greater mean total LR length than PRs and LGs, respectively (Fig. 3C). Mean LR length in PRs was 38\% greater than LGs (Fig. $3 \mathrm{C})$. When compared with SRs, the mean MR/LR length ratio was increased by $38 \%$ and $60 \%$ in PRs and LGs, respectively (Fig. 3D). The mean MR/LR length ratio among LGs was $38 \%$ greater than PRs. SR/LR mean surface area was $70 \%$ and $134 \%$ greater than PRs and LGs, respectively (Fig. 3E). PR/LR surface area was $37 \%$ greater than LG surface area. Relative to SRs, mean MR/LR surface area ratio was increased by $42 \%$ and $62 \%$ in PRs and LGs, respectively (Fig. 3F). The MR/LR surface area ratio among LGs was $35 \%$ greater than PRs. PCA showed that the first two principal components (PCs) accounted for $96 \%$ of the total variance (Table 1). PC1 accounted for $86 \%$ of the variance among samples with positive loadings of LR length, LR surface area, LR density, and LR count. 
PC2 accounted for an additional $11 \%$ of the variation with $L R$ count, MR/LR area ratio, LR density, and MR/LR length ratio loading positively. Proceeding from negative to positive values of $\mathrm{PC} 1$, it was evident that PRs classified as LGs and SRs occupied extreme locations with a gradient existing between these samples (Fig. 4A). Within this gradient, PRs represented an intermediate stage with significant overlapping from LGs and SRs. The extreme SR and LG specimens are shown in Figures $2 \mathrm{C}$ and $2 \mathrm{H}$, respectively. The PR specimen located at the extreme "bottom" of the horseshoe-shaped plot is shown in Figure 2E. The plot of PC1 and PC3 showed a general increase in scatter (variance) among PRs as well as the overlapping LGs and SRs on PC3 (Fig. 4B). This increased scatter among specimens reflects the fact that ARs with intermediate LR counts and MR/LR area ratios scored low on PC2.

Relationship between growth substrate moisture variability and lateral root development. Representative images of ARs representing shortest, intermediate, and longest total LR length for each of three moisture regimes (control, dry, saturated) 10 to 20 DAT from the total data set are shown in Figure 5. Relative to the control treatments, water deprivation 10 to 20 DAT reduced mean LR count, length, and surface area by $49 \%, 103 \%$, and $94 \%$, respectively (Figs. $6 \mathrm{~A}$, $6 \mathrm{C}$, and $6 \mathrm{E}$ ). Overwatering 10 to 20 DAT reduced LR mean count, length, and surface area by $86 \%, 81 \%$, and $77 \%$, respectively (Figs. 6A, 6C, and 6E). Relative to the control treatment, dry and overwatering regimes reduced mean LR density by $45 \%$ and $92 \%$, respectively (Fig. 6B). Relative to the control treatment, mean MR/LR length ratio was increased by $103 \%$ and $81 \%$ in dry and overwatering regimes, respectively. Mean MR LR surface area ratio was increased by $46 \%$ in dry and saturated conditions, respectively. There were no statistical differences for all AR attributes between the dry and saturated treatments.

\section{Discussion}

Root growth and architecture are important factors that influence plant performance and survival yet are frequently overlooked in horticultural research (Wright and Wright, 2004). Our results in part support the hypothesis, first suggested by Koshimizu and Nishida (1949) and reiterated by Pardales and Yamauchi (2003), that postulates a relationship between root system architecture and SR yield potential in sweetpotato. In general, ARs that showed evidence for SR initiation had higher values for all measured LRrelated attributes and lower values for calculated MR/LR-related ratios relative to PRs and LGs. PCA integrated these LR attributes and the "horseshoe"-shaped plot of PC1 and PC2 showed a gradient between the extreme LG and SR clusters with PRs appearing to represent an intermediate stage. Reyment (1991) has attributed the horseshoe pattern in PCA to a characteristic of data sets in which the correlations among variables are very high and approximately equal. The horseshoe pattern may also be caused by nonlinear data structures (Podani and Miklos, 2002). Hughes and Chapman (1995) observed a similar pattern in PCA of growth attributes in Silurian proetide trilobites (Aulacopleura Konincki) and attributed this pattern to the ontogenetic transition of growth mode of A. Konincki.
Wilson and Lowe (1973) referred to PRs as "intermediate structures" with some anomalous production of parenchyma cells in the central root axis. In 'Beauregard' sweetpotato, this horseshoe pattern appears to represent a growth trajectory where ARs with optimum LR number (Fig. 2E-F) but intermediate LR length possesses the competency to become SRs by undergoing further LR development On the other hand, ARs (Figs. 2D, 2G, and 2H) with low LR number and density do not appear capable of becoming SRs unless additional LRs develop. In Arabidopsis, Dubrovsky et al. (2006) have presented evidence that LR primordia development occurred strictly acropetally and no de novo initiation events were found between already developed LRs or LR primordia. Arrested or slowly developing LR primordia were found between developed LRs and it was concluded that there was a narrow development window for LR initiation (Dubrovsky et al., 2006). Thus, an elucidation of factors that influences LR number and density in sweetpotato will further enhance our knowledge of the presumptive association between root architecture plasticity and yield variability in this crop species. In the PCA plots, the overlapping of SRs and LGs indicate that LR development is not the sole determinant of SR formation. Current evidence shows that sucrose (Eguchi and Yoshida, 2008; Li and Zhang, 2003), cytokinin (Eguchi and Yoshida, 2008; Tanaka et al., 2008), and nitrogen (Villagarcia et al., 1998) are involved in the process of SR initiation. Our results provide a basis for further understanding how these variables influence SR initiation. Our data also provide evidence for the potential link between quantifiable morphological LR attributes

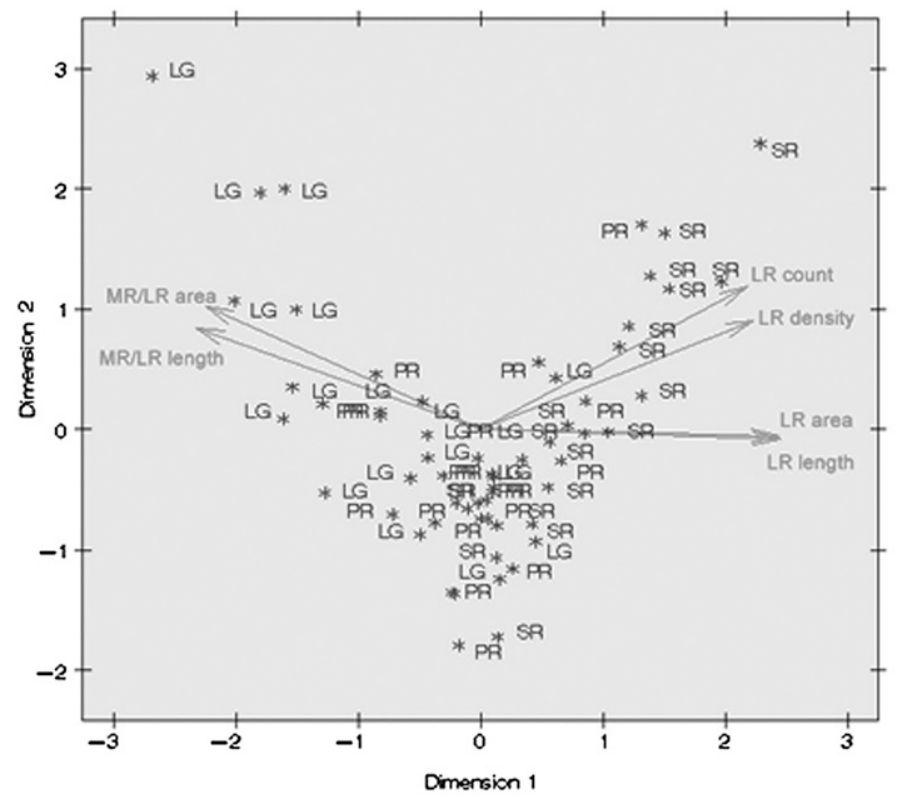

A

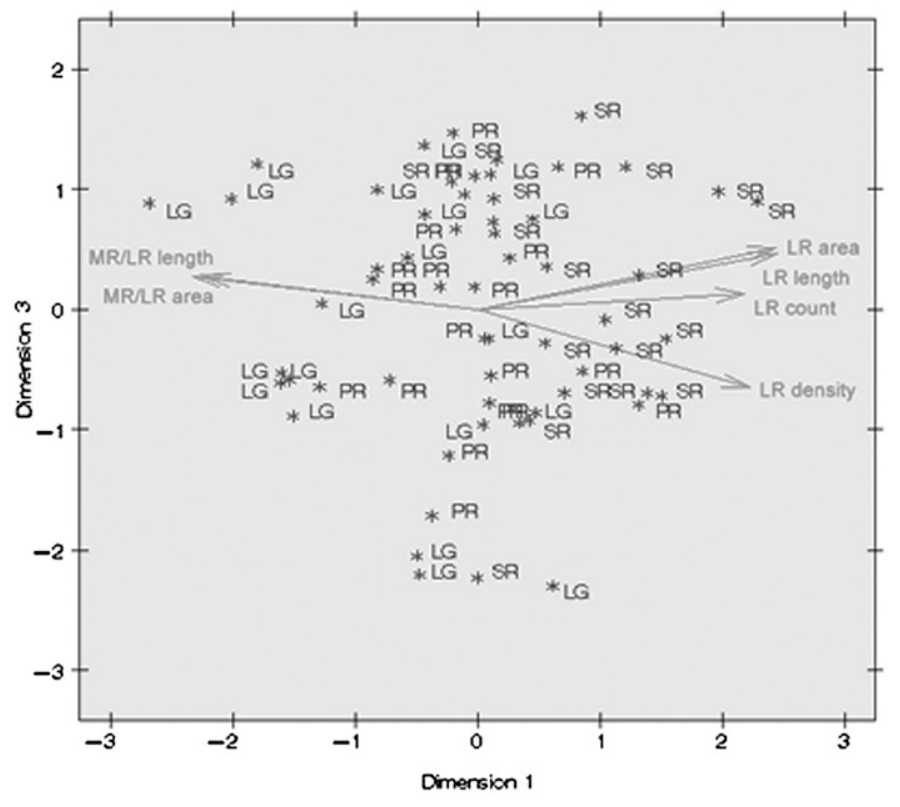

B

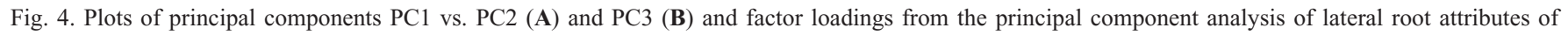
'Beauregard' adventitious roots. $\mathrm{LR}=$ lateral root; $\mathrm{MR}=$ main root; $\mathrm{MR} / \mathrm{LR}$ area $=\mathrm{MR} / \mathrm{LR}$ surface area ratio; MR/LR length = MR/LR length ratio; LR density $=$ LR number/main root length. $\mathrm{PC}=$ principal component. 


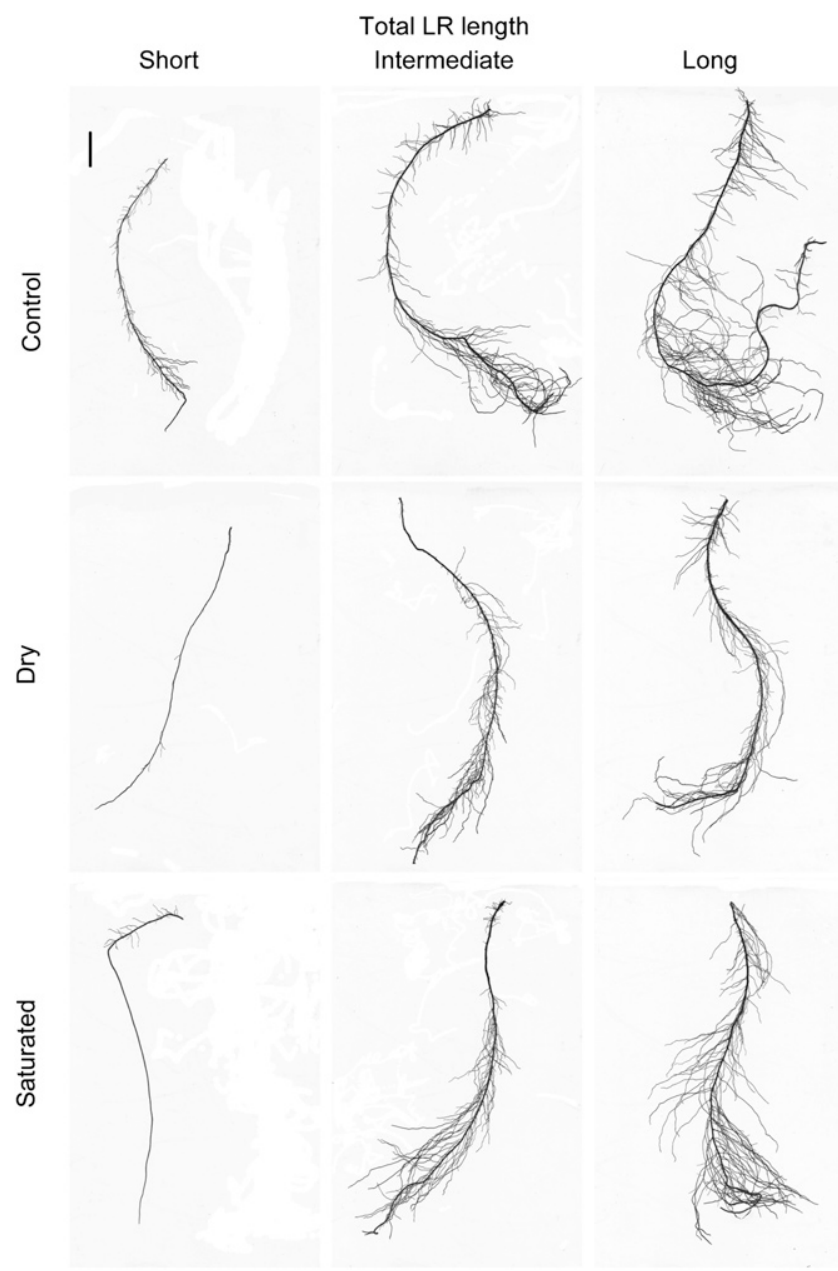

Fig. 5. Adventitious roots that represent shortest, intermediate, and longest cumulative lateral root length for each of the following growth substrate moisture regimes during the 10 - to 20 -d period in 'Beaureard' sweetpotato: control, dry, and saturated. Control = growth substrate moisture was maintained near $50 \%$ of field capacity (FC); Dry = no water after $10 \mathrm{~d}$ (moisture less than $25 \%$ of FC at harvest); Saturated $=$ moisture was maintained near $75 \%$ of FC. LR $=$ lateral root. Scale bar $=2 \mathrm{~cm}$

and an LR-specific molecular indicator of SR initiation as described by $\mathrm{Ku}$ et al. (2008).

In general, SRs had increased LR count, length, and surface area relative to LGs and PRs. This suggested a competitive advantage relative to other ARs in terms of access to soil moisture and nutrients. Green et al. (2005) used the concept of "interlateral root competition" in describing the reduction of soil volume exploited by developing Mediterranean shrub (Pistacia lentiscus) roots with shortened internode lengths. LR competition within and among plants may help to determine SR competency among ARs, especially in marginal environments. In the PCA plots, the gradient between extreme LG and SR samples indicated a quantitative component in the determination of the developmental fate of ARs, i.e., LG, PR, or SR. Morphological plasticity in response to competition effects has been investigated in sweetpotato. For example, Sulaiman and Sasaki (2001) observed a decrease of root and shoot dry weight per hill with increased planting densities among the sweetpotato genotypes that were studied and attributed this response to "intense interplant competition." In other species, LR plasticity has been documented to be involved in adaptive responses to the soil environment. For example, Borch et al. (1999) documented that low phosphorus availability reduced LR number and sustained MR elongation, resulting in decreased LR density in phosphorus-deficient common bean (Phaseolus vulgaris). Arredondo and Johnson (2011) proposed that the proliferation of fine roots deployed into enriched soil sites did not appear to require a precise placement of LRs but rather rapid initiation and elongation of multiple roots. In contrast, the appropriate strategy under marginal soil nutrient conditions appeared to involve the emergence of fewer and spatially distant roots that delayed LR competition and favored the improvement of root foraging precision (Berntson, 1994).

We demonstrated 'Beauregard' sweetpotato AR architecture plasticity in response to growth substrate moisture variability 10 to 20 DAT and provided corroborating data to an earlier report by Pardales and Yamauchi (2003). Under low and excessive moisture conditions 10 to 20 DAT, LR count, length, and surface area were significantly reduced in comparison with the control treatment. These data provide evidence that soil moisture variability during the first 20 DAT, i.e., optimum soil moisture followed by drought or saturated conditions, is directly related to the determination of yield potential in 'Beauregard' sweetpotato. This helps to explain earlier results reported by Togari (1950) about the yield-limiting effects of extreme soil moisture conditions during the first $20 \mathrm{~d}$. Pardales and Yamauchi (2003) reported variable and significant reductions in LR count ( $-44 \%$ to $-66 \%)$ and length ( $-59 \%$ to $-77 \%)$ at 28 DAT among sweetpotato genotypes subjected to continuous drought conditions. The magnitudes of these differences were similar to our results (Figs. 6A and 6C). Pardales and Yamauchi (2003) stated that drought was the "most limiting factor" in LR development during the "establishment stage," which they defined as the first 4 weeks of growth. However, our results showed that saturated conditions also hindered LR development within this timeframe. This helps to underscore the importance of properly calibrating irrigation frequency and amount for specific soil types in sweetpotato production. Our findings are also consistent with experimental results in other species. Seo and Park (2009) reported that drought conditions suppressed Arabidopsis LR development. Zook et al. (1986) have previously demonstrated that flooding conditions suppressed LR growth in alfalfa (Medicago sativa). In Arabidopsis, it has been shown that flooding conditions resulted in increased ethylene synthesis that inhibited lateral root initiation and root growth (Ivanchenko et al., 2008). This work can be used as a methodological model for conducting follow-up studies to determine if the sweetpotato root system possesses a similar mechanism for determining response to saturated conditions. Our findings can also be used to enhance current breeding and selection strategies, especially for low-input environments. For example, Zhu and Lynch (2004) documented that seedlings of five recombinant inbred lines of maize (Zea mays) had variable LR length responses under conditions of low phosphorus, a relatively immobile soil nutrient. Zhu and Lynch (2004) suggested that enhanced LR rooting under phosphorus stress can be harnessed as a useful trait for the selection and breeding of more phosphorus-efficient maize genotypes. Pardales and Yamauchi (2003) documented that imposition of optimum soil moisture conditions after dry or saturated conditions led to resumption of LR development and concluded that root architecture response to soil moisture variability can be manipulated not only for agronomic production, but also for breeding for stable production in environments subjected to soil moisture stress.

In model systems, it has been shown that LR initiation and development is mediated by internal cues and external variables. Internal cues of LR formation and development include auxin, ethylene, abscisic acid, and cytokinin (De Smet et al., 2003; Ivanchenko 

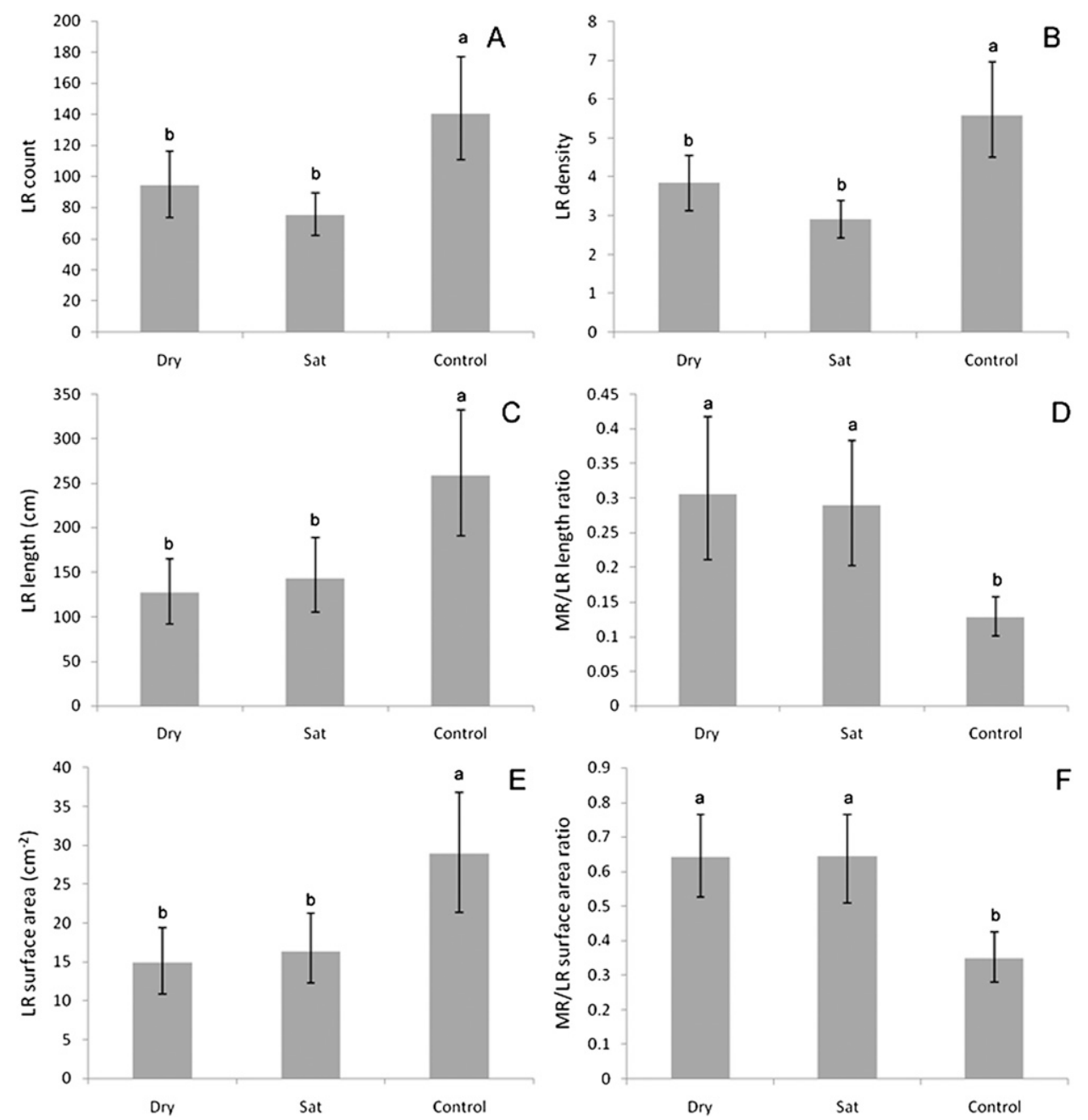

Fig. 6. Some statistical descriptive data of lateral root attributes of 'Beauregard' adventitious roots subjected to different growth substrate moisture regimes at 10 to $20 \mathrm{~d}$. Bars represent means \pm bootstrapped confidence intervals ( $95 \%$ confidence level). Mean comparisons were performed on transformed data: squaretransformed (measurements), $\log 10$ transformed (counts), and arcsine-transformed (ratios). Corresponding non-transformed values are shown. Columns with different letters differ significantly at the $5 \%$ level by Fisher's least significant difference. $L R=$ lateral root; MR = main root; Control = moisture was maintained near $50 \%$ of field capacity (FC); Dry = no water after $10 \mathrm{~d}$ (moisture less than $25 \%$ of FC at harvest); Saturated=moisture was maintained near $75 \%$ of FC.

et al., 2008; Lopez-Bucio et al., 2002). Environmental variables include soil nutrients such as nitrate (Zhang and Forde, 1998), phosphate (Johnson et al., 1996), and sulfate (Kutz et al., 2002). In many cases, nutrient variability appears to influence hormone sensitivity. For example, phosphorus deficiency alters Arabidopsis root architecture by changing sensitivity to auxins (LopezBucio et al., 2002). The information about the relationship between LR development and sweetpotato SR initiation helps to provide a basis for integrating the knowledge gained about internal and external cues that influence LR initiation and development in model systems.

\section{Conclusion}

We have documented the association between LR attributes and anatomical cues associated with onset of SR initiation in 'Beauregard' sweetpotato. We have also provided corroborating evidence that soil moisture variability 10 to 20 DAT directly influenced LR development and demonstrated the importance of optimizing soil moisture during the critical SR initiation period. Our data indicate that AR architecture is not the sole determinant of SR initiation. However, these findings can be used to integrate AR root architecture measurements with other variables that are known to influence SR initiation. This information can be used to further optimize management and environmental variables during the critical SR initiation period to further optimize SR yield in sweetpotato.

\section{Literature Cited}

Arredondo, J.T. and D.A. Johnson. 2011. Allometry of root branching and its relationship to root morphological and functional traits in three range grasses. J. Expt. Bot. 15:5581-5594.

Belehu, T., P.S. Hammes, and P.J. Robbertse. 2004. The origin and structure of adventitious roots in sweet potato (Ipomoea batatas). Aust. J. Bot. 52:551-558.
Berntson, G.M. 1994. Modelling root architecture: Are there tradeoffs between efficiency and potential of resource acquisition? New Phytol. 127:483-493.

Borch, K., T.J. Bouma, J.P. Lynch, and K.M. Brown. 1999. Ethylene: A regulator of root architectural responses to soil phosphorus availability. Plant Cell Environ. 22:425-431.

Casimiro, I., T. Beeckman, N. Graham, R. Bhalerao, H. Zhang, P. Casero, G. Sandberg, and M. Bennett. 2003. Dissecting Arabidopsis lateral root development. Trends Plant Sci. 8:165-171.

De Smet, I., L. Signora, T. Beeckman, D. Inze, C. Foyer, and H. Zhang. 2003. An abscisic acid-sensitive checkpoint in lateral root development of Arabidopsis. Plant J. 33:543555.

Dubrovsky, J.G., G.A. Gambetta, A. HernandezBarrera, S. Shishkova, and I. Gonzales. 2006. Lateral root initiation in Arabidopsis: Developmental window, spatial patterning, density and predictability. Ann. Bot. (Lond.) 97:903915.

Eguchi, T. and S. Yoshida. 2008. Effects of application of sucrose and cytokinin to roots on 
the formation of tuberous roots in sweetpotato (Ipomoea batatas L. Lam.). Plant Root 2:7-13.

Green, J.J., J.A. Baddeley, J. Cortina, and C.A. Watson. 2005. Root development in the Mediterranean shrub Pistacia lentiscus as affected by nursery treatments. J. Arid Environ. 61:112.

Hughes, N.C. and R.E. Chapman. 1995. Growth and variation in the Silurian proetide trilobite Aulacopleura konicki and its implications for trilobite paleobiology. Lethaia 28:333-353.

Ivanchenko, M., G. Muday, and J. Dubrovsky. 2008. Ethylene-auxin interactions regulate lateral root initiation and emergence in Arabidopsis thaliana. Plant J. 55:335-347.

Johnson, J.F., C.P. Vance, and D.L. Allan. 1996. Phosphorus deficiency in Lupinus albus. Altered lateral root development and enhanced expression of phosphoenolpyruvate carboxylase. Plant Physiol. 112:657-665.

Koshimizu, T. and M. Nishida. 1949. On the relation between the distribution of free-auxin in the young sweet potato plant and its roottuber formation. Bot. Mag. Tokyo. 62:735736.

$\mathrm{Ku}$, A.T., Y. Huang, Y. Wang, D. Ma, and K. Yeh. 2008. IbMADS1 (Ipomoea batatas MADSbox 1 gene) is involved in tuberous root initiation in sweet potato (Ipomoea batatas). Ann. Bot. (Lond.) 102:57-67.

Kutz, A., A. Muller, P. Henning, W.M. Kaiser, M. Piotrowsky, and E.W. Weiler. 2002. A role for nitrilase 3 in the regulation of root morphology in sulphur-starving Arabidopsis thaliana. Plant J. 30:95-106.

Laskowski, M.J., M.E. Williams, H.C. Nusbaum, and I.M. Sussex. 1995. Formation of lateral root meristems is a two-stage process. Development 121:3303-3310.
Li, X. and D. Zhang. 2003. Gene expression activity and pathway selection for sucrose metabolism in developing storage root of sweetpotato. Plant Cell Physiol. 44:630-636.

Lopez-Bucio, J., E. Hernandez-Abreu, L. SanchezCalderon, M.F Nieto-Jacobo, J. Simpson, and L. Herrera-Estrella. 2002. Phosphate availability alters architecture and causes changes in hormone sensitivity in the Arabidopsis root system. Plant Physiol. 129:244-256.

Pardales, J.R. and A. Yamauchi. 2003. Regulation of root development in sweetpotato and cassava by soil moisture during their establishment period. Plant Soil 255:201-208.

Podani, J. and I. Miklos. 2002. Resemblance and coefficients and the horseshoe effect in principal coordinate analysis. Ecology 83:33313343.

Reyment, R.A. 1991. Multidimensional palaeobiology. Pergamon, Oxford, UK.

Seo, P.J. and C. Park. 2009. Auxin homeostasis during lateral development under drought condition. Plant Signal. Behav. 4:1002-1004.

Sulaiman, H. and O. Sasaki. 2001. Studies on effect of planting density on the growth and yield of sweet potato (Ipomoea batatas Lam.). Mem. Fac. Agr. Kagoshima Univ. 37: $1-10$.

Tanaka, M., N. Kato, H. Nakayama, M. Nakatani, and Y. Takahata. 2008. Expression of class I knotted1-like homeobox genes in the storage roots of sweetpotato (Ipomoea batatas). J. Plant Physiol. 165:1726-1735.

Togari, Y. 1950. A study of tuberous root formation in sweet potato. Bul. Nat. Agr. Expt. Sta. Tokyo 68:1-96.

Villagarcia, M.R., W.W. Collins, and C.D. Raper, Jr. 1998. Nitrogen uptake and nitrogen use efficiency of two sweetpotato genotypes during early stages of storage root formation. J. Amer. Soc. Hort. Sci. 123:814-820.

Villordon, A., D. LaBonte, and J. Solis. 2011. Using a scanner-based minirhizotron system to characterize sweetpotato adventitious root development during the initial storage root bulking stage. HortScience 46:513-517.

Villordon, A.Q., D.R. LaBonte, N. Firon, Y. Kfir, E. Pressman, and A. Schwartz. 2009a. Characterization of adventitious root development in sweetpotato. HortScience 44:651-655.

Villordon, A., D.R. LaBonte, and N. Firon. 2009b. Development of a simple thermal time method for describing the onset of morpho-anatomical features related to sweetpotato storage root formation. Sci. Hort. 121:374-377.

Wilson, L.A. and S.B. Lowe. 1973. The anatomy of the root system in West Indian sweet potato [Ipomoea batatas (L.) Lam.] cultivars. Ann. Bot. (Lond.) 37:633-643.

Wright, A.N. and R.D. Wright. 2004. The Horhizotron: A new instrument for measuring root growth. HortTechnology 14:560-563.

Yasui, K. 1944. Notes on the propagation of sweet potato, Ipomoea batatas Lam. I. The adventives bud formation in the root-tuber. Proc. Imperial Acad. 20:41-44.

Zhang, H. and B. Forde. 1998. An Arabidopsis MADS box gene that controls nutrient-induced changes in root architecture. Science 279:407409.

Zhu, J. and J.P. Lynch. 2004. The contribution of lateral rooting to phosphorus acquisition efficiency in maize (Zea mays) seedlings. Funct. Plant Biol. 31:949-958.

Zook, D., D. Erwin, and L. Stolzy. 1986. Anatomical, morphological, and physiological responses of alfalfa to flooding. Plant Soil 96: 293-296. 